\title{
MEJORA DE LAS PROPIEDADES ADSORPTIVAS DE BIOMATERIALES MEDIANTE MODIFICACIONES QUÍMICAS EN LA ELIMINACIÓN DE ANTIBIÓTICOS
}

\author{
Jesie C. Silva ${ }^{\mathrm{a}}$, Lizebel Morante ${ }^{\mathrm{a}}$, Carlos J. Moreno ${ }^{\mathrm{a}}$, Norma A. Cuizano ${ }^{\mathrm{a}}$, \\ Abel E. Navarro*b, Bertha P. Llanos ${ }^{\mathrm{a}}$
}

\begin{abstract}
RESUMEN
Antibióticos y productos farmacéuticos se han convertido en contaminantes emergentes en aguas residuales debido al bajo control de su comercialización y distribución. En este proyecto se propone el uso de materiales de desecho de hojas de té de manzanilla (CM), té verde (GT) y menta (PM) y sus modificaciones químicas: carboxilación, tiolación y sulfonación como adsorbentes de Penicilina G. Las muestras se sometieron a reacciones químicas sostenibles, en medio acuosas y mínimas de reactivos. Los adsorbentes y sus modificaciones fueron caracterizados mediante técnicas instrumentales. Todos los adsorbentes reportaron estabilidad térmica constante y sólo ligeros cambios morfológicos de superficie. Las pruebas de adsorción demostraron una fuerte influencia de la acidez en la eliminación del antibiótico, sobre todo a nivel de la química de los nuevos grupos funcionales, destacando las interacciones dipolo-dipolo. Los porcentajes de adsorción indican que la tiolación y sulfonación fueron las modificaciones químicas más efectivas, sobre todo en la adsorbente $\mathrm{CM}$, alcanzando \%ADS de $27 \%$, dentro del rango de $\mathrm{pH}$ 7-8. La tendencia de máxima adsorción de Penicilina G tiene la secuencia: $\mathrm{CMs}>\mathrm{PMs} \geq \mathrm{GTs}$, mientras que las modificaciones químicas siguen la tendencia: Sulfonación $\geq$ Tiolación $>$ Carboxilación.
\end{abstract}

Palabras clave: Carboxilación, sulfonación, tiolación, hojas de té, antibióticos, adsorción.

a Sección Química, Departamento de Ciencias Exactas, Facultad de Ciencias y Filosofía, Universidad Peruana Cayetano Heredia, Av. Honorio Delgado 430, Lima 31, Perú.

b Science Department, Borough of Manhattan Community College, City University of New York, New York, NY, 10007, USA. anavarro@bmcc.cuny.edu ; 199 Chambers Street, N699H, New York, NY, 10007, USA 


\title{
ENHANCEMENT OF THE ADSORPTIVE PROPERTIES OF BIOMATERIALS BY CHEMICAL MODIFICATION FOR THE ELIMINATION OF ANTIBIOTICS
}

\begin{abstract}
Antibiotics and pharmaceutical products have become emergent contaminants in residual waters due to the inefficient commercialization and distribution. This study proposes the use of waste materials from tea leaves of chamomile (CM), green tea (GT) and peppermint (PM) and their chemical modifications: carboxylation, sulfonation and thiolation as adsorbents of Penicillin G. The tea samples were treated with sustainable chemical reactions in aqueous media and minimal reagent consumption. The adsorbents and their chemical modifications were characterized by instrumental techniques. All the adsorbents reported constant thermal stability and only small morphological changes on their surfaces. The adsorption tests demonstrated a strong influence of the acidity on the elimination of the antibiotic, mostly at the level of the chemistry of the added functional groups, mainly through dipole-dipole forces. The adsorption percentages indicate that thiolation and sulfonation are the most effective chemical modifications, highlighting $\mathrm{CM}$ adsorbents, reaching $\% \mathrm{ADS}$ of $27 \%$, within the $\mathrm{pH}$ range 7-8. The maximum adsorption tendency of Penicillin $\mathrm{G}$ has the sequence: $\mathrm{CMs}>$ PMs $\geq$ GTs, whereas the chemical modifications follow the trend: Sulfonation $\geq$ Thiolation $>$ Carboxylation.
\end{abstract}

Key words: Carboxylation, sulfonation, thiolation, tea leaves, antibiotics

\section{INTRODUCCIÓN}

Cada mañana, antes de acudir a nuestros centros laborales, desde que nos paramos frente a un espejo, usamos productos químicos. Sin darnos cuenta, contribuimos al vertido de estas sustancias a nuestras aguas superficiales. En las últimas décadas, la producción y consumo de productos del cuidado personal y farmacéuticos, tales como lociones, crema de manos, productos de higiene, pasta de dientes, lociones, aceites de protección solar, desinfectantes, repelentes de insectos y otros, se ha incrementado sustancialmente en los países desarrollados y más aún en los países en vías de desarrollo (dado el crecimiento poblacional más acelerado $)^{1}$. De acuerdo con los datos estadísticos internacionales del mercado farmacéutico global, se estimó haber producido medicamentos por valores de \$956 millones en el 2011 y esto incrementó a $\$ 1,2$ trillones en el 2013. Solo en los EE.UU. se estima que había 4 millones de prescripciones de medicamentos en el 2009 (ventas a $\$ 300$ millones). Además, en los EE.UU., se estima que hay 100000 medicamentos que no necesitan receta médica. Las ventas del mercado farmacéutico mundial por países fueron: USA $36 \%$, Europa 31,5 \%, Japón 11,3 \%, Asia (India, China, Korea), África (Sudáfrica, Nigeria, etc), Australia 12, 7 \%, Latinoamérica (Brasil, Argentina, etc) 5,7\% y Canadá 2,8 \% ${ }^{2,3}$. 
La aparición de los antibióticos en los ambientes acuáticos es un problema eco-toxicológico debido a la potencial alteración del ecosistema. La exposición prolongada a bajas dosis de antibióticos conduce a la proliferación selectiva de las bacterias resistentes, lo que podría transferir los genes de resistencia a otras especies bacterianas ${ }^{4}$. La contaminación de aguas superficiales y agua potable por antibióticos y otros compuestos farmacéuticos han sido reportados en estudios recientes a través de descargas desde sistemas de alcantarillado doméstico ${ }^{1}$.

La eliminación de antibióticos de aguas residuales depende principalmente de las etapas secundarias y procesos avanzados en el tratamiento, ya que los sólidos grandes son fácilmente eliminados por sedimentación. Generalmente, los efluentes de las aguas residuales son tratados por medio de la cloración o radiación ultravioleta; sin embargo, el tratamiento convencional en aguas residuales no está diseñado ni operado para eliminar concentraciones bajas de contaminantes, tales como fármacos, consecuentemente, estos son liberados hacia aguas superficiales ${ }^{4,5}$. En el Perú, el tratamiento de las aguas del río Rímac para la potabilización del agua es la siguiente: (1) captación, (2) desarenadores, (3) pre-cloración, (4) embalses reguladores, (5) dosificación de coagulantes en planta, (6) decantación, (7) filtración, (8) planta de recirculación de agua de lavado de filtros, (9) cloración y (10) reservorios de almacenamiento. En este proceso, estos contaminantes pueden seguir persistiendo durante años ya que sus concentraciones son bajas ${ }^{6}$. De esta manera, los métodos más utilizados son aquellos en que se emplean procesos de adsorción y procesos oxidativos avanzados; en este último se pueden formar productos intermedios como compuestos orgánicos halogenados donde se utiliza el cloro y ozono.

Un problema importante dentro de la categoría de antibióticos son las penicilinas, uno del grupo más importante de los antibióticos $\beta$-lactámicos. La penicilina se utiliza comúnmente como materia prima para la producción de penicilinas semi-sintéticas y son extremadamente eficaces y todavía se utilizan ampliamente en aplicaciones veterinarias y en medicina humana, de modo que su eliminación indiscriminada al medio ambiente podría eventualmente reducir su eficiencia en estos campos por el aumento de la resistencia de la penicilina a patógenos en humanos y animales?

Frente a este problema latente, la comunidad científica, encabezada por la biorremediación, se encuentra en la constante búsqueda de alternativas sostenibles y de bajo costo para eliminar contaminantes, ya sean orgánicos o inorgánicos ${ }^{8}$. La biosorción, como área de la biorremediación, es una tecnología efectiva para eliminar elementos metálicos y compuestos orgánicos de soluciones acuosas mediante el uso de materia no viviente. La biosorción involucra procesos fisicoquímicos basados en mecanismos complejos de reacción de adsorción, reacción de intercambio iónico con grupos funcionales en la superficie celular y acomplejamiento superficial ${ }^{8,9}$. A manera de comparación, existen otras tecnologías convencionales para eliminar trazas de contaminantes, como precipitación química, evaporación, galvanoplastia, adsorción e intercambio iónico, pero la biosorción tiene grandes ventajas como la alta eficiencia y bajo costo de la transformación de estos materiales en 
biosorbentes debido a que son económicos, entre ellos algas, hongos, lodos activados, etc. El uso de biomasa no viva evita problemas de contaminación, de igual manera que no afecta la cadena trófica porque se pueden regenerar ${ }^{8,9}$.

En el presente proyecto se investigó el uso de materiales de desecho (hojas de té) luego del proceso de infusión. Estas muestras constituyen una materia rica en carbohidratos, formado por una diversidad de grupos químicos funcionales tales como alcoholes, aminas, ésteres, ácidos carboxílicos, aldehídos, cetonas y otros grupos que se han caracterizado por su alta densidad electrónica y su capacidad de atraer centros positivamente cargados. Estos materiales ligno-celulósicos han sido utilizados previamente en la eliminación de metales pesados $^{10-12}$ y contaminantes organicos ${ }^{13-15}$ con resultados positivos. De acuerdos a estos estudios, la capacidad de adsorción de estos biomateriales reside en la presencia de grupos altamente polares como hidroxilo, carboxilo, amino y carbonilo que actúan como centros de atracción de los iones metálicos y de grupos polares de los contaminantes orgánicos ${ }^{10-15}$. Estos hallazgos sirvieron de premisa para este proyecto, en la búsqueda de la mejora de la adsorción mediante la sobrepoblación de los adsorbentes con estos grupos funcionales polares mediante la modificación química. Se propone que la carboxilación, sulfonación y tiolación de estos biomateriales conllevan una mayor afinidad de contaminantes.

Para este estudio se tomó como prioridad antibióticos, dada la importancia que han tomado en la Comunidad Europea y en la Agencia de Protección Ambiental de los EE.UU. ${ }^{8,16}$. Estos países sostienen que los antibióticos son compuestos de degradación que contribuyen al incremento de la resistencia bacteriana y por lo tanto inhiben la acción de los antibióticos actualmente disponibles. A largo plazo, nuestros antibióticos no serán efectivos, y las enfermedades serán más difíciles de combatir. Los antibióticos no solo son usados por humanos, sino también para uso veterinario. Por lo tanto, este proyecto se focaliza en el estudio de la eliminación del antibiótico Penicilina G (de uso humano). La estructura química de este antibiótico se muestra en la figura 1.

Dados nuestros resultados preliminares, decidimos empezar este proyecto de dos maneras: primero, la factibilidad de eliminar antibióticos por métodos de adsorción y segundo la optimización del proceso de adsorción mediante la modificación química de nuestros materiales de desecho de hojas de té de manzanilla (CM), té verde (GT) y menta (PM) que nos permita eliminar la mayor cantidad de antibiótico.

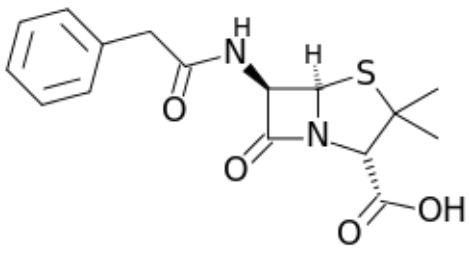

Figura 1. Estructuras químicas de Penicilina G 


\section{PARTE EXPERIMENTAL}

\section{Acondicionamiento y modificación química de los adsorbentes}

Se compró bolsas de té filtrante de manzanilla (CM), té verde (GT) y menta (PM) de un mercado local en Nueva York, EE.UU.

Esquema 1. Acondicionamiento de hojas de té

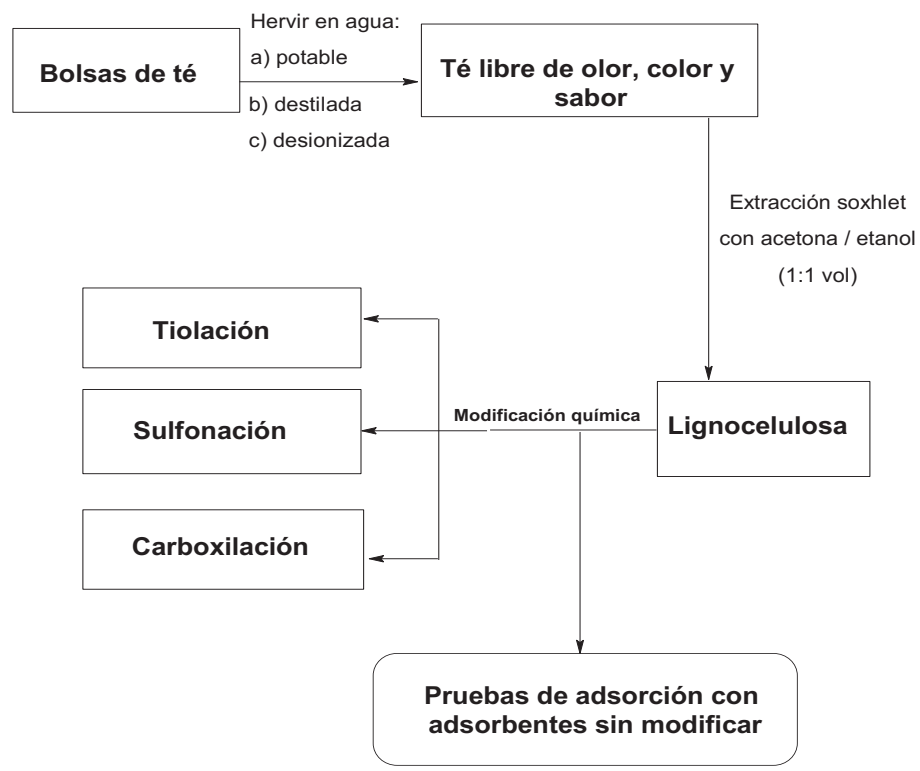

\section{Pre-tratamiento de las muestras antes de su modificación química}

Si bien la infusión en agua potable, destilada y desionizada asegura la liberación de impurezas de las muestras de té; sin embargo, existen residuos biológicos propios de las plantas como clorofila y tintes que son parte de la estructura de las mismas. Por lo tanto, fue necesario extraer estos compuestos poco solubles en agua que no fueron eliminados con agua caliente, para asegurarnos que la modificación química le ocurría exclusivamente a los polímeros ligno-celulósicos y no a la clorofila y/o tintes de las hojas.

Para ello, se condujo una extracción continua del tipo Soxhlet con una mezcla de acetona/ etanol. La extracción fue exitosa y se extrajo todo tinte. La nueva muestra se mostraba como pura celulosa y carbohidratos estructurales. La extracción en agua hirviente y por Soxhlet se muestra en la figura 2. Luego de la extracción Sohxlet, las muestras se secaron nuevamente en la estufa a la misma temperatura previamente reportada y luego almacenada en envases de vidrio hasta su uso en la modificación química. 

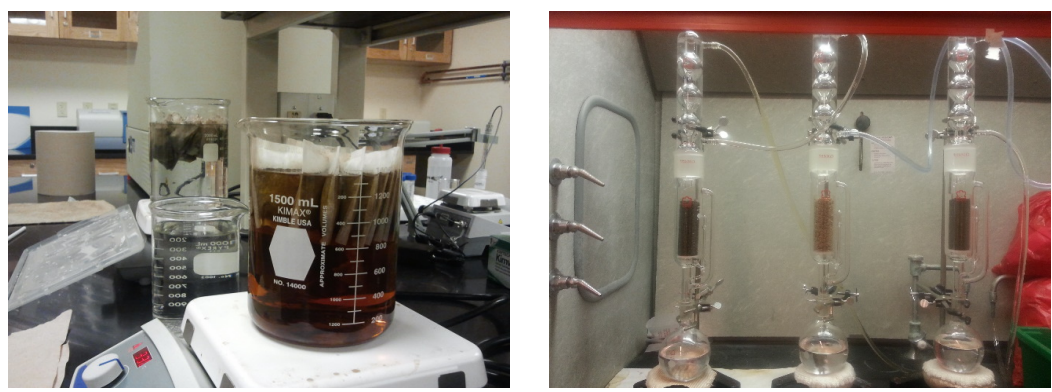

Figura 2. Extracción agua hervida (izquierda) y por el método Sohxlet (derecha)

\section{Modificaciones químicas de las muestras de té: Carboxilación, sulfonación y tiolación} Las muestras obtenidas del proceso Soxhlet fueron usadas para la modificación química. Los adsorbentes resultantes fueron designados de la siguiente manera: manzanilla carboxilada (CCM), manzanilla sulfonada (SCM) y manzanilla tiolada (TCM); menta carboxilada (CPM), menta sulfonada (SPM) y menta tiolada (TPM); té verde carboxilada (CGT), té verde sulfonada (SGT) y té verde tiolada (TGT).

Esquema 2. Sulfonación de las hojas de té acondicionadas ${ }^{17}$

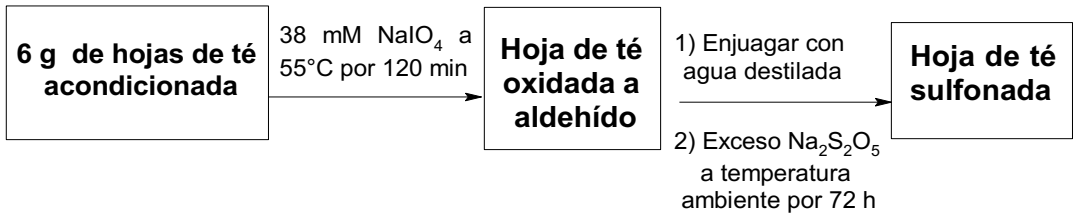

Para insertar grupos tioles, T, la biomasa se pre-trató en $300 \mathrm{~mL}$ de solución de $\mathrm{NaOH}$ al 1 $\%$ y $300 \mathrm{~mL}$ de etanol a temperatura ambiente $\left(25^{\circ} \mathrm{C}\right)$ durante $24 \mathrm{~h}$. Este proceso se utilizó para eliminar pigmentos de clorofila y otros compuestos de bajo peso molecular. Después de la decantación y filtración, la biomasa tratada se lavó doblemente con agua destilada hasta que la solución alcanzó un valor de $\mathrm{pH}$ neutro, y luego se secó a $70^{\circ} \mathrm{C}$ dentro de una estufa. A continuación, se agitó en $1000 \mathrm{~mL}$ de ácido mercapto-acético $\left(\mathrm{C}_{2} \mathrm{H}_{4} \mathrm{O}_{2} \mathrm{~S}\right)$ al $1 \%$ durante $12 \mathrm{~h}$ a temperatura ambiente $\left(25^{\circ} \mathrm{C}\right)$. Después de la decantación y filtración, el producto se lavó adicionalmente con agua destilada hasta que la solución alcanzó un valor de pH neutro. También se secó a $70{ }^{\circ} \mathrm{C}$ dentro de una estufa durante toda la noche ${ }^{18}$.

Finalmente, para insertar grupos carboxílicos, la biomasa se añadió a una solución de agua destilada con ácido cítrico e hipofosfito de sodio bajo agitación por 30 minutos a temperatura ambiente. Posteriormente, la suspensión se sometió a secado a $55^{\circ} \mathrm{C}$ y después de $24 \mathrm{~h}$ la temperatura se aumentó a $120^{\circ} \mathrm{C}$ por un intervalos de 30 a 110 minutos. Después se lavó con agua destilada tres veces y se secó a $55^{\circ} \mathrm{C}$ por $24 \mathrm{~h}^{19}$. 
Luego de las modificaciones químicas, las muestras fueron almacenadas en frascos de vidrio hasta su uso en las pruebas de adsorción y caracterización.

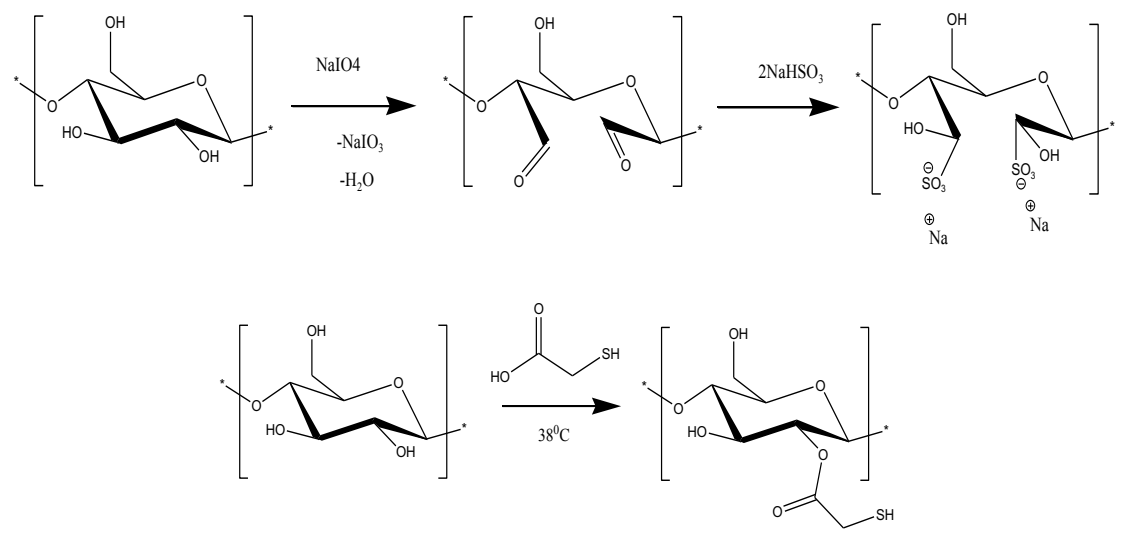

Figura 3. Reacciones químicas para la modificación de las muestras de té. (Arriba) sulfonación y (abajo) tiolación.

\section{Caracterización de las muestras de té nativo y químicamente modificado por técnicas clásicas e instrumentales}

La caracterización de los materiales nativos y modificados se realizó mediante técnicas instrumentales. La presencia de los grupos funcionales incorporados (carboxilo, sulfonilo y tiol) fueron corroborados por espectroscopia infrarroja. La textura y morfología de los adsorbentes antes y después de la modificación química fue estudiada mediante espectroscopia de barrido electrónico. Y, finalmente, la estabilidad y resistencia térmica y mecánica fue monitoreada por análisis termo-gravimétrico.

Los estudios de espectroscopia de infrarroja (FTIR) se realizaron con un equipo FTIR modelo Frontier de Perkin Elmer, con la ayuda de un accesorio de ATR para medir muestras en el estado sólido. Las microscopias de barrido electrónico se realizaron mediante el uso de un Scanning electron microscope (SEM) modelo TM3000 de Hitachi. No se necesitó la cubierta de oro y las muestras se estudiaron directamente en el instrumento. Finalmente, el análisis termo-gravimétrico (TGA) se llevó a cabo mediante un equipo modelo STA 6000 de Perkin Elmer. Las condiciones del experimento fueron en atmosfera de nitrógeno, a un flujo de calor de $5^{\circ} \mathrm{C} / \mathrm{min}$. Cada TGA requirió entre $7-10 \mathrm{mg}$ de muestra, en un rango de temperatura de $30-600^{\circ} \mathrm{C}$.

\section{Evaluación del efecto de la acidez en la adsorción de Penicilina G}

Para determinar el efecto del $\mathrm{pH}$ en la capacidad de adsorción de una determinada cantidad de adsorbente se preparó suficiente cantidad de solución del antibiótico de una determinada concentración (50ppm), se separaron en varios recipientes de plástico y cada uno se ajustó a pH de 2, 3, 4, 5, 6, 7 y 8, con soluciones diluidas de ácido clorhídrico e hidróxido de sodio. 
Luego, se pesaron 50mg de adsorbente y se agregó $50 \mathrm{~mL}$ de una solución ajustada a un determinado $\mathrm{pH}$, se repitió el mismo proceso para las otras soluciones de distintos $\mathrm{pH}$, los ensayos se realizaron por duplicado. Luego, estos se colocaron en un agitador a $250 \mathrm{rpm}$ durante 24 horas, tiempo suficiente para alcanzar el equilibrio. Al término de la agitación, se separaron las partículas de adsorbente de la solución mediante filtración y se procedió a determinar el contenido de penicilina $\mathrm{G}$ adsorbida por la técnica de espectrofotometría UVVisible, tanto para las soluciones remanentes como de los blancos a una longitud de onda de $220 \mathrm{~nm}$.

\section{Procesamiento de los datos experimentales}

La cuantificación de Penicilina G se expresó en porcentaje de adsorción (\%ADS) de acuerdo a la ecuación 1:

$$
\% A D S=\frac{\left(\mathbf{c}_{\mathbf{i}}-\mathbf{c}_{e q}\right) * 100}{\mathbf{c}_{\mathbf{i}}}
$$

donde Ci y Ceq son las concentraciones iniciales y al equilibrio de Penicilina en las soluciones. El tratamiento matemático y estadístico se realizó mediante los paquetes Microsoft Office, EXCEL y el paquete estadístico ORIGIN, de OriginLab. Se calcularon las desviaciones estándar de las pruebas de adsorción y se encontraron por debajo del $5 \%$ de error para todos los experimentos. Barras de error no se añadieron a los gráficos para evitar congestionamiento de la imagen.

\section{RESULTADOS Y DISCUSIÓN}

Las muestras de té nativas y modificadas fueron preparadas y no existieron problemas técnicos ni de resistencia del material frente a las condiciones de las reacciones químicas. Hubo cierta pérdida de masa de los adsorbentes antes y después de la modificación química de alrededor del 15-20\% de la masa inicial, pero esta pérdida era predecible dada la extracción Sohxlet y el tratamiento de la lignocelulosa en medio acido (para todas las modificaciones químicas).

\section{Análisis de grupos funcionales por espectroscopia de infrarrojo (FTIR)}

Se realizaron espectros de FTIR de todas las muestras nativas y sus modificaciones químicas. Parte de los resultados para la manzanilla y derivados se muestra en la figura 4. De los resultados se evidencia cambios en los grupos funcionales de la manzanilla. Por ejemplo, se evidencia cambios sustanciales en la intensidad del pico alrededor de 3300 (presencia de grupos hidroxilo - alcohol). CCM reporta el pico más intenso, debido al aumento de grupos carboxilo $\mathrm{COOH}$, que debe incrementar la señal de $\mathrm{OH}$ en el FTIR. Por otro lado, SCM y TCM muestran un acortamiento de la señal del -OH a 3300. Esto se justifica debido a la reducción de grupos alcohol $(-\mathrm{OH})$ para permitir la incorporación de $-\mathrm{SH}$ y $-\mathrm{SO}_{3} \mathrm{H}$. También cabe resaltar la disminución de la intensidad de los picos - $\mathrm{CH}$ sp3 alrededor de 2950 para los adsorbentes modificados. Este cambio se podría sustentar por la hidrolisis parcial de ciertos esteres (que poseen grupos alquilo) durante las modificaciones químicas. Recordemos que la modificación química es en condiciones acidas, lo cual promueve la pérdida (hidrolisis) de esteres, causando pérdida de grupos alquilo, y por ende reducción de la señal en 2950. 
Lamentablemente, los grupos tiol y sulfonilo no son claramente hallados en el espectro FTIR debido a que sus picos aparecen en la región del "fingerprint", la cual es muy saturada de picos, que impiden una determinación exacta. Sin embargo, los cambios en los grupos $-\mathrm{OH}$, evidencian que hubo un cambio en la estructura. Resultados similares se obtuvieron para las mentas y té verdes. Se podría concluir de estos resultados que las tres modificaciones químicas sucedieron para todos los adsorbentes.

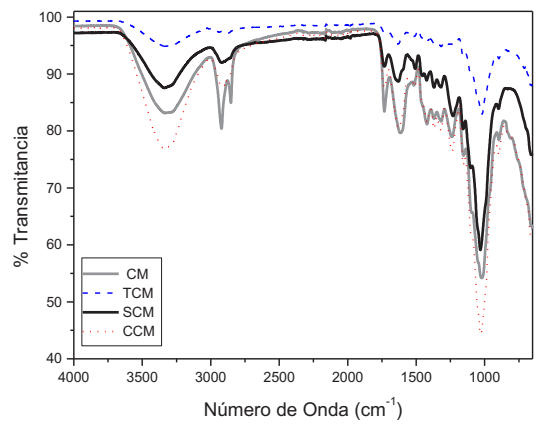

Figura 4. Espectros FTIR de la manzanilla nativa y sus modificaciones químicas.

\section{Microscopia de barrido electrónico}

La textura y análisis morfológico de las muestras se estudió mediante microscopia de barrido electrónico. De acuerdo a los resultados (figura 5), se muestran superficies heterogéneas en todos los adsorbentes; evidenciando su potencial uso como adsorbentes. La presencia de poros y rugosidades en la superficie indica que el antibiótico puede quedarse atrapado en esas imperfecciones, facilitando la interacción de grupos funcionales del adsorbente. La figura 5 solo muestra las microscopias de GT y derivados; sin embargo, todas las muestras mostraron resultados muy similares.
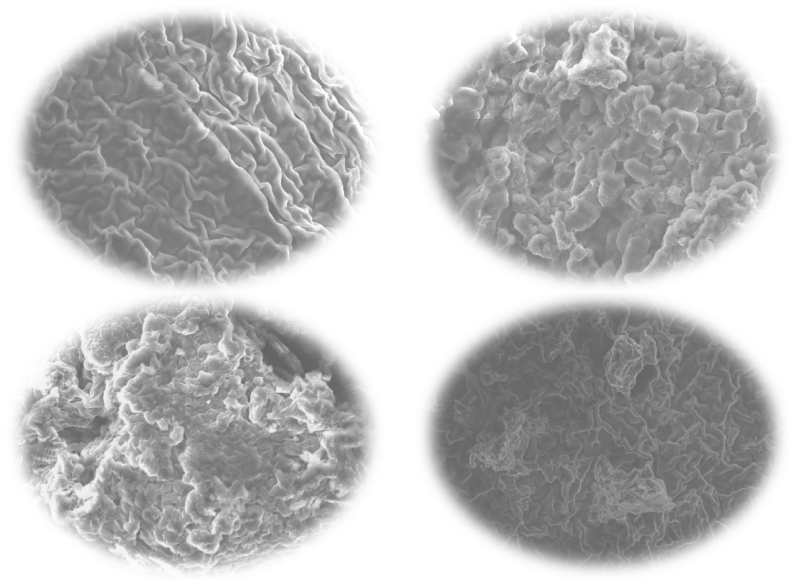

Figura 5. Microscopias de barrido electrónico (SEM) de las muestras de té verde (superior izquierda), té verde carboxilado (superior derecha), té verde sulfonado (inferior izquierdo) y té verde tiolado (inferior derecho). 
Cabe destacar de los resultados de la figura 5 que hay cambios sustanciales y esperando, debido a las modificaciones químicas. Debido al tratamiento acido en cada una de las reacciones, se observan cambios en la superficie. Si bien las superficies se mantienen heterogéneas, pero hay cierto deterioro de las mismas. Este efecto es más intenso en la tiolación, y la más leve en la carboxilación. Esto puede tener un aspecto favorable en sentido de la continuidad del proyecto. Ya que estos cambios estructurales de las muestras de té, evidencian que la lignina es parcialmente degrada. Esta degradación sería de importante aplicabilidad en materia de producción de bio-combustibles. Estudios científicos aseguran que la producción de biocombustibles, a partir de materiales lignocelulósicos, está limitado por la presencia de la rígida estructura de lignina. Dado que nuestros adsorbentes modificados muestran cambios estructurales, se podría plantear la hipótesis que la lignina ha sido parcialmente degrada, y podría facilitar la generación de bio-combustibles. Por lo tanto, el beneficio sería doble, las modificaciones químicas incrementan la adsorción de antibióticos en las hojas de té, y además favorecen la producción de bio-combustibles.
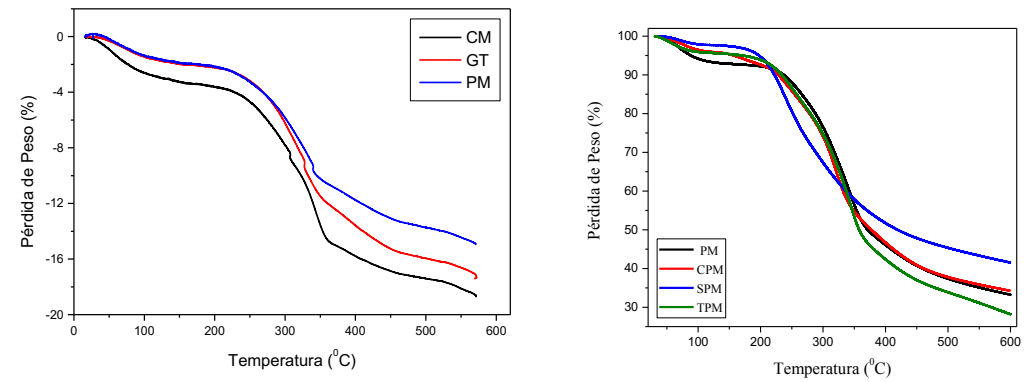

Figura 6. Análisis termo-gravimétricos de las muestras de té nativo (izquierda) y de las modificaciones químicas de menta (derecha).

\section{Análisis termo-gravimétrico}

Un adsorbente ideal debe ser capaz de tener resistencia térmica y mecánica para poder resistir situaciones adversas como temperatura y medios agresivos como oxidantes, ácidos y humedad. Esta resistividad puede medirse en función de la estabilidad térmica. Mientras más resistente a la temperatura es un material, más estable y fuertes son las estructuras y polímeros que forman su esqueleto. Bajo esta premisa, se estudió el análisis termo-gravimétrico de todas las muestras. En la figura 6 (izquierda) se muestran los TGA de los té nativos, indicando una mayor estabilidad para GT y PM, comparado con la manzanilla, CM. Este resultado era predecible, dado que $\mathrm{CM}$ es en realidad una flor y por lo tanto más frágil en sentido de composición de polímeros estructurales. Se podría concluir que la menor rigidez polimérica de CM propicia una mejor incorporación de los grupos funcionales. GT y PM son más robustos y por lo tanto más difíciles de modificar químicamente. Por otro lado, las modificaciones químicas de la menta, PM se muestran también en la figura 6 (derecha). De los resultados, se concluye que la tiolación y carboxilación tienen efectos en la resistencia de la muestra, pero no muy sustanciales. Al contrario, se podría decir que la carboxilación aumenta, ligeramente, la estabilidad de la muestra. Este resultado podría explicarse dado el estado de oxidación del grupo carboxilo. Este grupo no puede oxidarse más, porque representa el máximo estado de 
oxidación de una muestra orgánica, por lo tanto, la acción del calor no tienen un efecto tan fuerte como en muestras que sí pueden oxidarse (nativo, tiolado o sulfonado). En conclusión, la modificación química tiene un efecto mínimo en la resistencia mecánica y térmica de los adsorbentes nativos, indicando que estos materiales aun retienen su integridad estructural. Si bien SEM indica que hay cambios estructurales (de acuerdo a las microscopias), sin embargo los polímeros estructurales de celulosa y hemicelulosa podrían permanecer intactos.
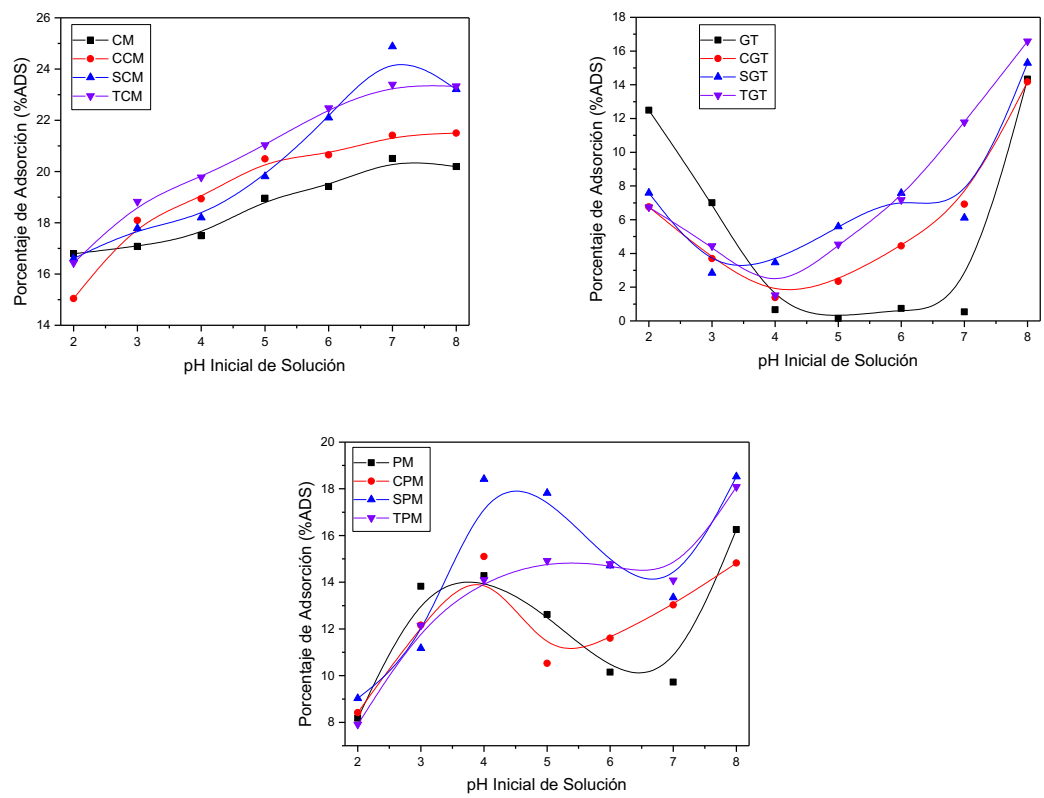

Figura 7. Rol del pH inicial de solución en la adsorción de Penicilina G en los adsorbentes nativos y modificados.

\section{Rol de la acidez en la adsorción de Penicilina G}

Luego de la preparación de las muestras de té, estos nuevos materiales se probaron como adsorbentes potenciales del antibiótico Penicilina G, a partir de soluciones acuosas bajo agitación orbital de $250 \mathrm{rpm}$ a temperatura ambiente. La concentración inicial y final se midió mediante espectrofotometría visible. Los resultados preliminares de la adsorción de Penicilina $G$ se muestran en la figura 7 para cada uno de los grupos de adsorbentes. De las gráficas, se demuestra que todas las modificaciones químicas causan un aumento de la adsorción de Penicilina G a las condiciones experimentales trabajadas. Este estudio comparó las muestras nativas con las modificaciones, mostrando una gran variabilidad con el pH inicial de la solución. Sin embargo, la adsorción del antibiótico sigue la tendencia: $\mathrm{CMs}>\mathrm{PMs} \geq$ GTs. Aparentemente, los polisacáridos estructurales de la familia de las manzanillas (CMs) muestran una mayor afinidad con Penicilina G. Como se mencionó anteriormente, GT y PM son hojas de planta, mientras que CM es la flor de una planta. Esta característica podría explicar las diferencias en la adsorción. En cuanto a las diferencias entre las modificaciones químicas, 
se observa que la sulfonación y tiolación presentan la mayor adsorción para todos los tipos de adsorbentes. Se podría postular que la presencia de azufre y formación de dipolo-dipolo aumenta la afinidad de Penicilina G. Cabe resaltar que de acuerdo a la figura 1, Penicilina G presenta azufre dentro de su estructura, por lo tanto la formación de puentes disulfuro entre el antibiótico y los grupos sulfonilo y tiol, bajo condiciones oxidantes, no puede ser descartada. Sin embargo, se necesitan más experimentos para probar esta hipótesis. Por otro lado, la carboxilación de las muestras no generó cambios sustanciales en la adsorción de Penicilina G. Este fenómeno podría explicarse en base a la formación de puentes de hidrógeno internos entre los grupos carboxilo. En conclusión, las modificaciones químicas siguen la tendencia: Sulfonación $\geq$ Tiolación $>$ Carboxilación.

La química del antibiótico también mostró efectos de la acidez de la solución. Por ejemplo las muestras $\mathrm{CMs}$ tienen un comportamiento lineal de la adsorción a $\mathrm{pH}$ crecientes con una máxima adsorción a $\mathrm{pH} 7$, mientras que los GTs muestran adsorciones máximas a $\mathrm{pH} 2$ y 8. Las muestras PMs tienen un comportamiento más heterogéneo en respuesta al $\mathrm{pH}$, con máxima adsorción a valores de 4 y 8 . En conclusión, el rol de la acidez en la adsorción de Penicilina $\mathrm{G}$ reside primordialmente en la química acuosa de los grupos funcionales y las muestras de adsorbentes, mas no en la química acuosa del antibiótico. Además, la máxima adsorción observada para todos los adsorbentes se ajusta a condiciones reales de aguas residuales, dentro del rango de $\mathrm{pH}$ 7-8. En la actualidad se están llevando a cabo más pruebas de adsorción para optimizar la adsorción con los adsorbentes modificados óptimos a diferentes condiciones experimentales de masa de adsorbente, concentración de antibiótico, salinidad, presencia de detergentes, presencia de otros contaminantes y tiempo.

\section{CONCLUSIONES}

El problema de la eliminación de contaminantes emergentes como antibióticos y otros productos farmacéuticos está cobrando auge en el área de la biotecnología. La biosorción ha sido empleada en el tratamiento de aguas residuales en las últimas décadas, focalizándose en la remediación de metales pesados. Sin embargo, la complexidad química de los nuevos contaminantes emergentes requiere nuevas directrices para la optimización de la adsorción. En este proyecto se usaron residuos de hojas de té de manzanilla (CM), té verde (GT) y menta (PM) y sus modificaciones químicas (carboxilación, tiolación y sulfonación) para la remoción de Penicilina $\mathrm{G}$ a partir de soluciones acuosas. Se realizaron las modificaciones químicas en medio acuoso, con la minimización de reactivos y la máxima incorporación de nuevos grupos funcionales. Los adsorbentes producidos se caracterizaron mediante espectroscopia infrarrojo, microscopia de barrido electrónico, y termo-gravimetría. Los resultados indican que los adsorbentes modificados incorporaron los nuevos grupos químicos, retienen las propiedades térmicas y morfológicas, adecuadas para biosorbentes. Se observaron cambios en la superficie de los productos tiolados, principalmente. La resistencia térmica indica que la presencia de polisacáridos estructurales de las muestras nativas no fue afectada a consecuencia de la reacción química. Las pruebas de adsorción demuestran que la tiolación y sulfonación fueron las modificaciones químicas más efectivas, sobre todo en 
la adsorbente $\mathrm{CM}$, alcanzando \%ADS de $27 \%$, dentro del rango de $\mathrm{pH} 7-8$. La tendencia de máxima adsorción de Penicilina G tiene la secuencia: $\mathrm{CMs}>\mathrm{PMs} \geq \mathrm{GTs}$, mientras que las modificaciones químicas siguen la tendencia: Sulfonación $\geq$ Tiolación $>$ Carboxilación.

\section{AGRADECIMIENTO}

Este proyecto fue financiado por INNOVATE-Perú, proyecto 169-PNICP-ECIP-2015 y a C3IRG grant \#1224 de la City University of New York. Se agradece a las instalaciones de la Sección Química de la Facultad de Ciencias y Filosofía de la UPCH por la ayuda brindada durante la ejecución de los experimentos.

\section{REFERENCIAS BIBLIOGRÁFICAS}

1. Silva J. Biosorción de Penicilina G como Contaminante emergente con adsorbentes naturales y químicamente modificados. [Tesis de Licenciatura]. Lima, Perú: Universidad Peruana Cayetano Heredia; 2016.

2. Mottaleb MA, Meziani MJ, Matin MA, Arafat MM, Wahab MA. ACS Symposium Series. Vol 1198, USA: ACS; 2015, p. 43-74.

3. Center for Disease Dynamics, Economy and Policy. State of the World's Antibiotics. CDDEP. Washington DC; 2015, p. 26-32.

4. Jjemba P. Excretion and Ecotoxicity of Pharmaceutical and Personal Care Products in the Environment. Ecotoxicol Environ Saf. 2006;63(1):113-130.

5. Ternes T, Joss A. Human Pharmaceuticals, Hormones and Fragrances: The Micropollutant Challenge for Urban Water Management. London: IWA Publishing; 2007.

6. Cunningham V, Buzby M, Hutchinson T, Mastrocco F, Parke N, Roden N. Effects of Human Pharmaceuticals on Aquatic Life: Next Steps. Environ Sci Technol. 2006; 40(11):3456-3462.

7. Bush K. Antimicrobial Agents. Curr Opin Chem Biol. 1997; 1(2): 169-175.

8. Liu Y, Wang J. Fundamentals and Applications of Biosorption Isotherms, Kinetics and Thermodynamics. New York: Nova Science Publishers; 2009.

9. Kotrba P, Machova M, Macek T. Microbial biosorption of metals. New York: Ed. Springer Science; 2011.

10. Choi Y, Isaac P, Irkakhujaev S, Masud ME, Navarro AE. Use of spent tea wastes-chitosan capsules for the removal of divalent copper ions. J J Environ Sci. 2015; 1(1): 003.

11. Navarro AE, Musaev H, Serrano K, Masud ME. Adsorption kinetics of cobalt (II) ions onto alginate beads from aqueous solutions. J Earth Sci Climate Change. 2014; 5:223. doi: 10.4172/2157-7617.1000223.

12. Park R, Kim G, Shen L, Hong M, Navarro AE. Batch adsorption of heavy metals onto chai tea residues for the bioremediation of contaminated solutions. Curr Top Biotechnol. 2014; 8: 51-62. 
13. Zarzar A, Hong M, Llanos B, Navarro AE. Insights into the eco-friendly adsorption of caffeine from contaminated solutions by using hydrogel beads. J Environ Anal Chem 2:150. doi: 10.4172/2380-2391.1000150.

14. Japhe T, Zhdanova K, Rodenburg L, Roberson L, Navarro AE. Factors affecting the Biosorption of 2-Chlorophenol using spent tea leaf wastes as adsorbents. J J Environ Sci. 2015; 1(2): 010.

15. Zahir H, Naidoo M, Kostadinova RM, Ortiz KA, Sun-Kou R, Navarro AE. Decolorization of hair dye by lignocellulosic waste materials from contaminated waters. Front Environ Toxicol. 2014, 2:28. doi: 10.3389/fenvs.2014.00028

16. Japhe T, Paulsingh R, Ko K, Hong J, Navarro AE. Bioremoval of antibiotics by using biodegradable hydrogel beads from aqueous solutions. J J Environ Sci. 2015; 1(1):002.

17. Liimatainen H, Visanko M, Sirvio J, Hormi O, Niinimaki J. Sulfonated cellulose nanofibrils obtained from wood pulp through regioselective oxidative bisulfite pretreatment. Cellulose. 2013; 20:741-749.

18. Wu Z, Cheng Z, Ma W. Adsorption of $\mathrm{Pb}$ (II) from glucose solution on thiol-functionalized cellulosic biomass. Bioresour. Technol. 2012; 104: 807-809.

19. Wang S, Wang L, Kong W, Ren J, Liu C, Wang K, Sun R, She D. Preparation, characterization of carboxylated bamboo fibers and their adsorption for lead(II) ions in aqueous solution. Cellulose. 2013; 20:2091-2100. 